\title{
Taking a poke at the liver: which way is best?
}

\section{(c) $(1)$}

\author{
Authors \\ Neil D. Shah, Todd H. Baron \\ Institution \\ Division of Gastroenterology and Hepatology, University of \\ North Carolina at Chapel Hill School of Medicine, Chapel \\ Hill, North Carolina, United States \\ Bibliography \\ DOI https://doi.org/10.1055/a-0747-5713 | \\ Endoscopy International Open 2019; 07: E72-E73
}

Percutaneous liver biopsy was described as early as 1923 [1] and today it still plays a pivotal role in management of patients with liver disease. Liver biopsy became more widely used in the 1960 s with the "one-second" technique described by Menghini in which he coupled suction from a syringe with a quick jab of a needle to obtain adequate samples of liver tissue, a concept that is still used today [2]. Over the last five decades, there has been evolution in liver biopsy. Imaging such as transabdominal ultrasound and computed tomography guidance can target specific lesions, help avoid vital structures, and help decrease procedure-related pain. Development of transjugular liver biopsy via interventional radiologic techniques is an accepted alternative in instances where percutaneous techniques are contraindicated, such as in patients with coagulopathy, ascites or morbid obesity.

More recently, endoscopic ultrasound-guided liver biopsy (EUS-LB) has emerged as an effective option for liver biopsy acquisition. EUS-LB offers many advantages over other methods such as real-time Doppler assistance to avoid vascular structures and eliminate the need for patient cooperation with inspiration/exhalation. Multiple studies have shown the efficacy of EUS-LB with diagnostic yields ranging from $91 \%$ to $100 \%$ and with very low adverse event (AE) rates [3-7]. EUS-LB also appears to have similar diagnostic yield to liver biopsies obtained from transjugular or percutaneous routes, which makes it attractive as another tool for hepatologists [3]. However, the current pitfall with EUS-LB is the variability that exists in both technique and standards of diagnostic adequacy.

A variety of commercially available fine-needle aspiration (FNA) and fine-needle biopsy (FNB) needles can be used for EUS-LB and they vary in their cutting mechanisms. Furthermore, a wide range of needle gauges from 14G to $20 \mathrm{G}$ have been used to perform EUS-LB. Other factors include the technique of tissue acquisition which has included use of wet suction, a "slow pull" technique, fanning and number of "to and fro" passes. Standards of adequacy also vary in the literature. Per the American Association for the Study of Liver Disease (AASLD), parameters for adequacy of liver samples is defined as number of complete portal triads (CPTs) $\geq 11$ and total specimen length (TSL) $\geq 30 \mathrm{~mm}$ [8]. Other standards have also been suggested for adequacy including CPTs $>6$ and TSL $>15 \mathrm{~mm}$ [9].

A few studies have provided some insight into which needles or techniques may provide adequate tissue. Schulman et al. evaluated six needle types in human cadaveric liver, including FNA, FNB and percutaneous needles, and concluded that one 19G FNB needle provided superior diagnostic yield [10]. Another study also showed that a "slow pull" technique had higher yield than standard suction technique in pancreatic samples [11]. In this issue of Endoscopy International Open, Mok et al. add to the literature to help determine optimal tissue acquisition in EUS-LB by performing the first in vivo, randomized, prospective study comparing a 19G FNA needle to a 22G FNB needle.

In this study, 20 patients underwent EUS-LB, were randomized to liver biopsy of a lobe by one needle (19G FNA or 22G FNB), and then underwent repeat liver biopsy of the same lobe with the other needles in crossover fashion. Both lobes of the liver were biopsied for all study patients, resulting in a total of 80 biopsies. Using adequacy defined as $\geq 5$ CPTs, there were more adequate samples in the 19G FNA group compared to the $22 \mathrm{G}$ FNB group ( $80 \%$ vs $53 \%, P=0.03$ ). However, interestingly, the number of CPTs per needle was 7.4 for the 19G FNA samples and 6.1 for the 22G FNB samples, which was not statistically different. The authors also discovered increased postprocessing fragmentation of the 22G FNB samples as compared to the 19G FNA samples, which they believe led to the discrepancy in sample adequacy. Similar to other studies of EUS-LB, there were no serious AEs.

The study helps to highlight that needle type plays an important role when it comes to tissue adequacy for diagnostic pur- 
poses. However, the study design may have flawed the results. The authors recognize and discuss several of these limitations. The comparison of two different types of needles (FNA vs. FNB) as well as two different gauges makes it difficult to explain the origins of any significant differences. Furthermore, the difference in needles made it impossible for the endoscopist to be blinded to the needle during the procedure, which can introduce bias. However, the authors bring up an important consideration for EUS-LB, which is fragmentation due to tissue processing. The $22 \mathrm{G}$ samples consistently had more fragmentation which influences pathologic diagnoses such as fibrosis, inflammation and steatosis. Fragmentation is not routinely reported in EUS-LB studies, but we believe should be an important parameter when considering the best method for EUS-LB tissue acquisition.

Despite some of the limitations of the current study, it helps shed light on the issue of needle type in EUS-LB by showing that a 22G FNB needle may not provide tissue adequacy compared to a 19G FNA needle. The rate of adequacy in this study for the $19 G$ needle mimics the results in the current literature, therefore, it is likely that the needle gauge itself led to decreased diagnostic yield in the current study. Based on this, it is likely that a needle gauge of 19G or larger should be used for EUS-LB in order to obtain more complete cores that have greater numbers of CPTs. However, future studies are still needed to determine whether FNA or FNB needles have higher diagnostic yield. Other questions also remain including the optimal number of passes, which lobe of the liver (or both) to sample, and whether suction increases yield.

In addition, studies are needed to help determine when blood is obtained versus true tissue cores, which can be difficult with gross inspection. Future, prospective studies will be needed to help answer some of these issues to optimize EUS-LB. Other challenges still include the practicality or cost-effectiveness of EUS-LB compared to other routes. But currently EUS-LB should be considered as a novel alternative for procuring hepatic tissue for chronic liver disease in patients that are already undergoing endoscopic evaluation or for those in whom a percutaneous or transjugular approach fails or is contraindicated.
Competing interests

Todd Baron is consultant and speaker for BSCl, Cook Endoscopy, Olympus and Medtronic.

\section{References}

[1] Bingel A. Ueber die parenchympunktion der leber. Verh Dtsch Ges Inn Med 1923; 35: 210-212

[2] Menghini G. One-second needle biopsy of the liver. Gastroenterology 1958; 35: $190-199$

[3] Pineda J], Diehl DL, Miao CL et al. EUS-guided liver biopsy provides diagnostic samples comparable with those via the percutaneous or transjugular route. Gastrointest Endosc 2016; 83: 360 - 365

[4] Parekh PJ, Majithia R, Diehl DL et al. Endoscopic ultrasound-guided liver biopsy. Endosc Ultrasound 2015; 4: 85 - 91

[5] Sey MS, Al-Haddad M, Imperiale TF et al. EUS-guided liver biopsy for parenchymal disease: a comparison of diagnostic yield between two core biopsy needles. Gastrointest Endosc 2016; 83: 347 - 352

[6] DeWitt J, Cho CM, Lin J et al. Comparison of EUS-guided tissue acquisition using two different 19-gauge core biopsy needles: a multicenter, prospective, randomized, and blinded study. Endosc Int Open 2015; 3: E471 -E478

[7] Nieto J, Khaleel H, Challita Y et al. EUS-guided fine-needle core liver biopsy sampling using a novel 19-gauge needle with modified 1-pass, 1 actuation wet suction technique. Gastrointest Endosc 2018; 87: $469-475$

[8] Rockey DC, Caldwell SH, Goodman ZD et al. American Association for the Study of Liver Diseases. Liver biopsy. Hepatology 2009; 49: 1017 1044

[9] Bravo AA, Sheth SG, Chopra S. Liver biopsy. N Engl J Med 2001; 344: $495-500$

[10] Schulman AR, Thompson CC, Odze R et al. Optimizing EUS-guided liver biopsy sampling: comprehensive assessment of needle types and tissue acquisition techniques. Gastrointest Endosc 2017; 85: 419426

[11] Lee JM, Lee HS, Hyun JJ et al. Slow-pull using a fanning technique is more useful than the standard suction technique in EUS-guided fine needle aspiration in pancreatic masses. Gut Liver 2018; 12: 360 - 366 